\title{
Reducing Size, Weight, and Power (SWaP) of Perception Systems in Small Autonomous Aerial Systems
}

\author{
Kennie H. Jones ${ }^{1}$ \\ NASA Langley Research Center, Hampton, VA, 23681 \\ and \\ Jason Gross $^{2}$ \\ West Virginia University, Morgantown WV 26501
}

\begin{abstract}
The objectives are to examine recent trends in the reduction of size, weight, and power (SWaP) requirements of sensor systems for environmental perception and to explore new technology that may overcome limitations in current systems. Improving perception systems to facilitate situation awareness is critical in the move to introduce increasing autonomy in aerial systems. Whether the autonomy is in the current state-of-the-art of increasing automation or is enabling cognitive decisions that facilitate adaptive behavior, collection of environmental information and fusion of that information into knowledge that can direct actuation is imperative to decisions resulting in appropriate behavior. Artificial sensory systems such as cameras, radar, LIDAR, and acoustic sensors have been in use on aircraft for many years but, due to the large size and weight of the airplane and electrical power made available through powerful engines, the SWaP requirements of these sensors was inconsequential. With the proliferation of Remote Piloted Vehicles (RPV), the trend is in significant reduction in SWaP of the vehicles. This requires at least an equivalent reduction in SWaP for the sensory systems. A survey of some currently available sensor systems and changing technology will reveal the trend toward reduction of SWaP of these systems and will predict future reductions. A new technology will be introduced that provides an example of a desirable new trend. A new device replaces multiple conventional sensory devices facilitating synchronization, localization, altimetry, collision avoidance, terrain mapping, and data communication in a single integrated, small form-factor, extremely lightweight, and low power device that it is practical for integration into small autonomous vehicles and can facilitate cooperative behavior. The technology is based on Ultra WideBand (UWB) radio using short pulses of energy rather than continuous sine waves. The characteristics of UWB yield several desirable characteristics to facilitate integration of perception for autonomous activities. The capabilities of this device and its limitations will be assessed.
\end{abstract}

\section{Introduction}

$\mathrm{W}$ hile unmanned and Remotely Controlled (RC) vehicles date back to the late $19^{\text {th }}$ century and radio-controlled RC airplanes were demonstrated as early as the $1940 \mathrm{~s}^{1}$, new technology has driven an unprecedented surge in the availability, use, and application of RC, autonomous, and otherwise unmanned vehicles in ground, aerial, and marine applications. Industry trade publications are dominated by descriptions of new vehicle designs, supporting technology, and applications. Articles appear regularly in nonprofessional publications such as newspapers, magazines, and other periodicals describing a new world in which autonomous vehicles will offload many humancentric tasks. Military applications have been the major driver as the desire to reduce risk to humans and cost of operation for surveillance, ordinance detection, supply delivery, and weapon delivery has rapidly increased commensurate with developing technology.

Recently, potential civil Unmanned Aerial Systems (UAS) applications have gained much attention in a wide variety of applications ranging from precision agriculture, delivery services (e.g., Amazon) ${ }^{2}$, science applications

\footnotetext{
${ }^{1}$ Senior Computer Engineer, Electromagnetics and Sensors Branch, Address/Mail Stop, AIAA Senior Member.

${ }^{2}$ Assistant Professor, Department of Mechanical and Aerospace Engineering, AIAA Member.
}

1

American Institute of Aeronautics and Astronautics 
such as remote sample return (e.g., water sampling) ${ }^{3}$, remote sensing of volcano plumes ${ }^{4}$, search, rescue and disaster relief $^{5}$, and commercial aerial surveillance used in real estate, movies and television, and by Hollywood paparazzi ${ }^{6}$. For these applications, the much lower costs associated with small, mini, or even micro Low Altitude Short Endurance (LASE) UASs make them most attractive. Continued research and development is required to enable UAS integration in the National Air Space (NAS), and particular challenges arise when considering low SWaP UAS systems. To facilitate identifying and prioritizing the challenges associated with integrating UAS into the national airspace, the FAA has published a Civil UAS roadmap ${ }^{7}$. The roadmap identifies the critical functional areas that will require additional research and development, as the state-of-the-art has been deemed too immature in comparison to manned systems, of which Sense and Avoid (SAA) technologies are emphasized.

The size of RC and autonomous UAVs varies considerably. The use of full sized vehicles is exemplified by Lockheed Martin's adaptation of the Kaman K-MAX Helicopter ${ }^{8}$ for non-line-of-sight RC and autonomous operation. Kaman's K-MAX helicopter was originally designed as a piloted external lift helicopter for heavy loads. Its empty weight is $5,145 \mathrm{lbs}$. with a maximum takeoff weight of 12,000 lbs. Under contract to the U.S. Army Aviation Applied Technology Directorate's Autonomous Technologies for Unmanned Air Systems (ATUAS) demonstration program, the Kaman K-MAX external-lift helicopter was adapted with advanced capabilities for RC and autonomous operation. To assist remote pilots when the vehicle is out of sight, a high-definition video is sent over high-bandwidth satellite communications providing improved situational awareness for the operator. The vehicle navigates autonomously using GPS to follow waypoints to a specified location. It includes dynamic mission replanning capability. If given a no-fly zone, the aircraft automatically replans a route around it. To facilitate autonomous landing, a three-dimensional laser radar develop by Fairchild Controls, the Hellas LIDAR ${ }^{9}$, was utilized to avoid obstacles and select a landing-zone, autonomously checking the landing zone slope and detecting obstacles to select a safe area for dropping the cargo. As an example of sensor SWaP on the K-MAX, the Hellas LIDAR occupies $1.1 \mathrm{ft}^{3}$, weighs $50.7 \mathrm{lbs}$., and consumes $330 \mathrm{~W}$ at peak consumption.

Perhaps the most successful UAS to date has been the Predator ${ }^{10}$. Originally designed and built for reconnaissance and forward observation, it has been adapted for many diverse missions. It has an empty weight of 1,130 lbs., a maximum takeoff weight of 2,250 lbs., and a payload of $450 \mathrm{lbs}$. It is noteworthy that, while small SWaP is always desirable and advantageous, vehicles in classes such as the K-MAX and Predator can accommodate sensor and perception payloads of considerable SWaP and many are available.

However, the trend is for significantly smaller vehicles ${ }^{11}$ ranging from tens of lbs. down to the eventual goal of emulating small birds or even insects. One consideration is that small size vehicles can more easily be transported and managed in the field. Another is performance, as many applications (e.g., surveillance) require no payload beyond the onboard sensors. Thus, the smaller vehicle is cheaper, requires less fuel, etc. In the case of reconnaissance and surveillance, the smaller the vehicle, the less likely it will be detected. As competition increases to make ever smaller vehicles, the SWaP requirements of sensors and perception systems become paramount.

Smaller vehicles require smaller SWaP for component sensor and perception systems. However, the need for better perception systems transcends SWaP issues. Immediately after their invention, the power of using electronic computers to control machines for autonomous operation was recognized. But this has only recently become pervasive with the advent of microelectronics. Since the invention of microprocessors and the development of Micro Electro-Mechanical Systems (MEMS), development of controllers for many kinds of electro-mechanical actuation has rapidly accelerated and embedded computers are now appearing in every type of machine. Industry has become very sophisticated at automatically controlling the action of machines. What has not progressed as rapidly is the ability to properly assess the environment to decide what action should be taken. This must be improved to move from automated machines to truly autonomous machines: those that can adapt to changing conditions. In order to completely remove the need for a remote monitoring crew and provide a truly autonomous vehicle that can adapt to an underdetermined or undetermined and changing environment, an autonomous vehicle must be capable of complete situation management facilitated by situation awareness made possible by multiple sensors inputting to perception systems that can fuse sensor input and feed a cognitive decision engine to determine correct action resulting in acceptable and desirable behavior.

Such perception systems must have a comprehensive environmental view, with a variety of and redundant sensing capabilities. Replacing the functioning pilot with machines provides the potential to not only replace human perception but to surpass it. As stated, for small UAS, these perception systems must not only be capable and accurate but must also be small in SWaP. At this point, both goals of perceptive capability and small SWaP may be viewed as in their infancy. It is not a goal here to provide solutions for these goals but to mark trends of some needed perceptive technologies in the provision of adequate capability and accuracy in a small SWaP format. In Section II, a brief introduction will be presented of some of these technologies. In Section III, a new technology will

2

American Institute of Aeronautics and Astronautics 
be presented in more detail that provides an intriguing combination of sensor capability in a small SWaP format. Section IV will present conclusions and recommendations for future investigation.

\section{Survey of Available Technologies}

In order to replace all onboard and remote pilots, an autonomous vehicle must be able to emulate human perception. If the autonomous vehicle is small, then the perception system must be small. The purpose here is to survey available small SWaP technologies that can be applied to the emulation of human perception. Although many factors are sensed and considered by pilots when deciding action for flight, only a few basic functions will be considered: how to perceive current position and orientation, how to track motion, how to navigate, and how to avoid obstacles.

The challenges of overcoming the technological hurdles of SAA become even more complex when considering the airworthiness of low SWaP UAS systems. The safety requirements are no less important and the potential number of these platforms for civil applications is greater than their larger counterparts, yet, the resources available for employing high precision state-of-the-art sensor technologies are much less, effectively ruling out many of the technologies. Toward addressing this challenge, academic and industry research has been conducted in the area technologies for application in UAS. Included here are passive cameras, Global Navigation Satellite Systems (GNSS), Inertial Navigation Systems (INS), and LIDAR for rangefinding and image mapping.

\section{A. Cameras}

Recent technological improvements in digital photography have produced extremely low SWaP, high performance visible spectrum cameras as evidenced by inclusion of multiple cameras in devices as small as cell phones. Thus, SWaP is not a problem for use of cameras onboard but much research is needed to go beyond capture of high definition photographs and video to emulate human analysis of the images. One optical navigation and collision avoidance approach inspired by insects has been a very active research direction for integration in UAS is extracting Optical Flow (OF) fields from cameras. OF provides a field representation of UAS velocity and distance/velocity of the surrounding environment ${ }^{12}$, which has been shown to provide an accurate passive navigation aide $^{13}$ for fusion with other small SWaP navigation sensors. Vision based SAA have shown the promise of outperforming human pilot performance and exceeding FAA guidelines ${ }^{14}$ under certain conditions. However, these approaches are sensitive to lighting and atmospheric conditions. Without incorporating specific dynamic maneuvers and interrupting the flight mission, no range estimation of objects is available with vision only approaches. Therefore, current techniques require that these must be coupled with LIDAR or RADAR.

\section{B. GNSS}

Global Navigation Satellite System (GNSS) is a generic term for satellite navigation systems that provide autonomous geo-spatial positioning. The U.S. Global Positioning System (GPS) provides global coverage, is widely used, and very small SWaP receivers are available (e.g., cell phones and smaller devices include GPS receivers). However, use of GPS stretches the definition of autonomy, as it requires unobstructed line of sight (LOS) to four or more GPS satellites (i.e., infrastructure outside of the vehicle is required). This introduces the possibility of failure beyond the capability of the vehicle and is a reality if LOS is lost (e.g., under some type of canopy or inside a cave or building). Further, an important cause for concern for solely relying on GPS or other satellite based positioning systems within safety critical systems such as autonomous UAS flight, are their susceptibility to jamming and spoofing ${ }^{15}$. Recent demonstrations by Todd Humphrey and his research group at University at Texas Austin have shown a sophisticated GPS spoofer's ability to hijack a UAS and a luxury yacht ${ }^{16}$.

Accuracy may also be a problem for use of GPS in navigation and SAA of closely cooperating vehicles. Depending upon conditions and equipment (e.g. single or dual-frequency, mult-constellation receivers), conventional GPS is accurate to within 3 to $15 \mathrm{~m}$. This can be substantially augmented with additional techniques and infrastructure. Two orders of magnitude accuracy improvement over conventional GPS (from $5 \mathrm{~m}$ to $5 \mathrm{~cm}$ ) is available from a Real-Time Kinematic (RTK) GPS receiver. However, RTK requires an additional burden of a fixed base station of known location. An RTK receiver from Sokkia, the GRX2 ${ }^{17}$, is available but it is relatively expensive, over a $1 \mathrm{~kg}$. in weight, $18 \mathrm{~cm} \mathrm{x} 10 \mathrm{~cm}$ in size, and requires a $20 \mathrm{gm}$., $5240 \mathrm{mAh}$ battery to operate for 7.5 hrs. Swift Navigation has recently released the Piksi ${ }^{18}$ RTK GPS receiver. Providing $50 \mathrm{~Hz}$ position/velocity/time solutions, the Piksi is a $53 \mathrm{~mm}$ square board weighing $32 \mathrm{~g}$. and consuming $500 \mathrm{~mW}$. With such small SWaP, the Piksi is promising for small UAS. 
Differential GNSS augmentation services that have global coverage and are not tied to the need a reference station also exist for achieving decimeter level real-time positioning accuracy. These systems are based on the Precise Point Positioning (PPP) technique ${ }^{19}$, and use a global network of tracking stations to determine precise orbit and clock corrections, which are then broadcast to the end user. For example, the Jet Propulsion Laboratory's Global Differential GPS System (GDGPS) has broadcast differential corrections to locations over the globe, including veryhigh latitude flights, using an Iridium link to support NASA JPL's Uninhabited Aerial Vehicle Synthetic Aperture Radar (UAVSAR) instrument ${ }^{20}$. Commercial global augmentation services are also available, such as NavCom's StarFire service $^{21}$, OmniStar $^{22}$, and Septentrio's TERRASTAR-D ${ }^{23}$. These typically rely on a link to a geostationary satellite (e.g. Inmarsat) and are often broadcast close to the GPS L-band to facilitate using the same antenna for GPS and the corrections signal. These systems rely on the use geodetic quality GNSS receivers, which still have a small SWaP profile. For example, Novatel's OEM-628 receiver is $60 \times 100 \times 9.1 \mathrm{~mm}$ and weighs $37 \mathrm{~g}$ can consumes $1.3 \mathrm{~W}$ with offering a $100 \mathrm{~Hz}$ update rate.

\section{Inertial Navigation Systems}

Significant SWaP reduction in inertial sensing technologies has occurred over the past decades driven largely U.S. Department of Defense's desire to become less dependent on GPS. In particular, DARPA has had programs invested in drastically increasing the accuracy of small SWaP MEMS inertial sensors and more recently cold atom technologies for small SWaP inertial sensing ${ }^{24}$. Throughout this period, the accuracy of MEMS consumer-grade inertial sensors has steadily increased, however, to-date, these sensors require fusion with an external aiding source (e.g. GPS) to compensate for sensor bias and drift and be useful for navigation purposes. While R\&D efforts are trending toward the goal of self-sufficient small SWaP inertial navigation systems for applications such as small SWaP UAS navigation, the current state-of-the-art requires fusion with other navigation sources.

A popular series of MEMS interial sensors are Analog Devices Intelligent Sensor series ${ }^{25}$. For example, the ADIS-16488, is very low SWaP with a size of 47 × 44 × $14 \mathrm{~mm}$, a weight of only 48 grams, and less than $1 \mathrm{~W}$ power consumption. This sensor suite also offers magnetometers and an on-board MEMS barometer for additional navigation aiding and reports an Angular Random Walk of $0.3 \% / \sqrt{ } \mathrm{hr}$ and an In-run Stability of $0.3 \% / \mathrm{hr}$. An earlier version of this IMU, the ADIS-16434, has been evaluated using UAV flight data for attitude estimation accuracy alongside a direct pitch and roll 'truth' measurements from high-quality mechanical vertical gyroscope and shown capable of real-time attitude estimates within a few degreed accuracy when coupled with GPS sensors ${ }^{26}$.

SBG systems provided a combination package, the IG-500 OEM ${ }^{27}$ that provides a small and accurate Attitude and Heading Reference System (AHRS) by combining a complete MEMS based Inertial Measurement Unit (IMU) with a Extended Kalman Filter that provides an accurate and drift-free 3D orientation. It coordinates its IMU with a GPS receiver and a barometric altimeter supporting navigation by providing a precise 3D orientation, velocity and position at high update rate up to $100 \mathrm{~Hz}$. It also accepts various external aiding information to improve precision for both attitude and position measurements. Without its aluminum enclosure, it weighs less than $10 \mathrm{~g}$. on a board

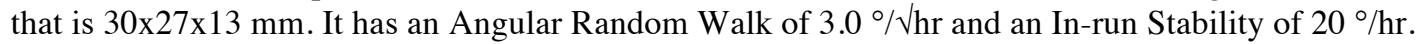

Gladiator Technologies accommodates an interesting strategy. They provide the Landmark series, a variety of IMUs of increasing accuracy and $\operatorname{cost}^{28}$. They provide a software infrastructure so that units may be easily upgraded at higher cost if more accuracy is required. There is also a choice of SWaP as the units range from $16 \mathrm{~cm}^{3}$ to 321 $\mathrm{cm}^{3}, 28 \mathrm{~g}$ to $388 \mathrm{~g}$, and $330 \mathrm{~mW}$ to $2200 \mathrm{~mW}$. The Landmark 40 is comparable to the ADIS-16488 as it has an Angular Random Walk of $0.24 \% / \sqrt{\mathrm{hr}}$ and an In-run Stability of $6.0 \% / \mathrm{hr}$, size of $50 \times 45$ x $32 \mathrm{~mm}$, weight of $103 \mathrm{~g}$, and power consumption of $430 \mathrm{~mW}$.

Epson provides an extremely small IMU, the $\mathrm{V} 340^{29}$. With a volume of only $0.48 \mathrm{~cm} 3$, it weighs $1 \mathrm{~g}$, and consumes $18 \mathrm{~mA}$ of current. The accuracy is comparable to others: Angular Random Walk is $0.20 \% \sqrt{\mathrm{hr}}$ and In-run Bias Stability is $7^{\circ} / \mathrm{hr}$.

\section{LIDAR for Rangefinding and Image Mapping}

3-D navigation can be enabled or enhanced with LIght Detection and Ranging (LIDAR) systems by three methods: laser rangefinders, Time of Flight (TOF) flash LADAR cameras, and scanning LIDAR.

Laser rangefinder sensors project a laser beam onto a target and receives some percentage of the reflection. The time required to receive the reflection (TOF) multiplied by the speed of light renders the distance from the laser to the target. Due to divergence of the laser beam, the distance cannot be great (typical $<1 \mathrm{~km}$ ) and atmospheric conditions and other environmental factors may exacerbate distortion. Due to the high speed of light, these devices are inaccurate for sub-meter distances. An obvious limitation to this approach is that a target must be preidentified and the device must be aimed at the target. 
FLIR provides an assortment of small SWaP rangefinders. Their MLR- $100^{30}$ miniature laser rangefinder is designed for determining distances in fast moving objects or vehicles. The MLR-100 model has a range capability better than $100 \mathrm{~m}$ at a resolution better than $20 \mathrm{~cm}$. Typical applications include altimetry or collision avoidance and other fast response proximity sensing. Its size is under $3 \mathrm{in}^{3}$, it weighs $22 \mathrm{~g}$, and consumes under $400 \mathrm{~mW}$.

An excellent use of a laser rangefinder is as an altimeter and West Virginia University (WVU) has implemented Opti-Logic's RS400 industrial laser rangefinder ${ }^{31}$ for this purpose. The unit weighs less than $8 \mathrm{oz}$ and consumes 1.8 $\mathrm{W}$ of power and has a reported accuracy of $+/-1$ meter with 0.2 -meter resolution over a range of 3.65 to 365 meters. This sensor has been flight-tested on WVU's Phastball research $U_{A S}^{32}$, where it is mounted downward looking (see Fig 1).

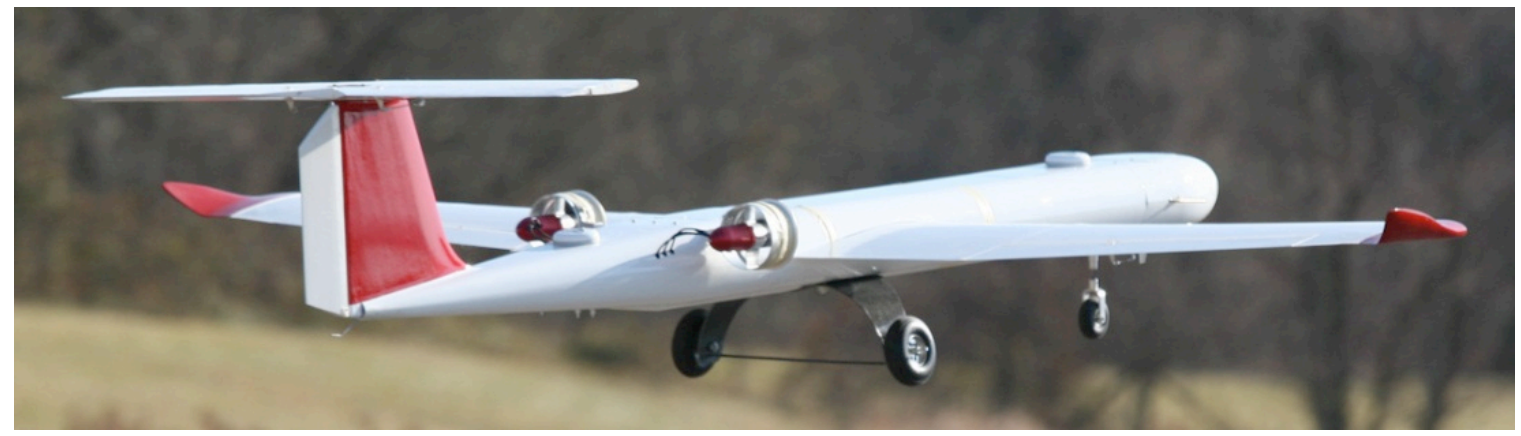

Figure 1. WVU Phastball Research UAS

A comparison of the laser range measurement and single frequency GPS receiver altitude solution after compensating for aircraft pitch and roll rotations on the laser range measurements shown in Fig. 2 (top) as well as the difference between them (bottom).
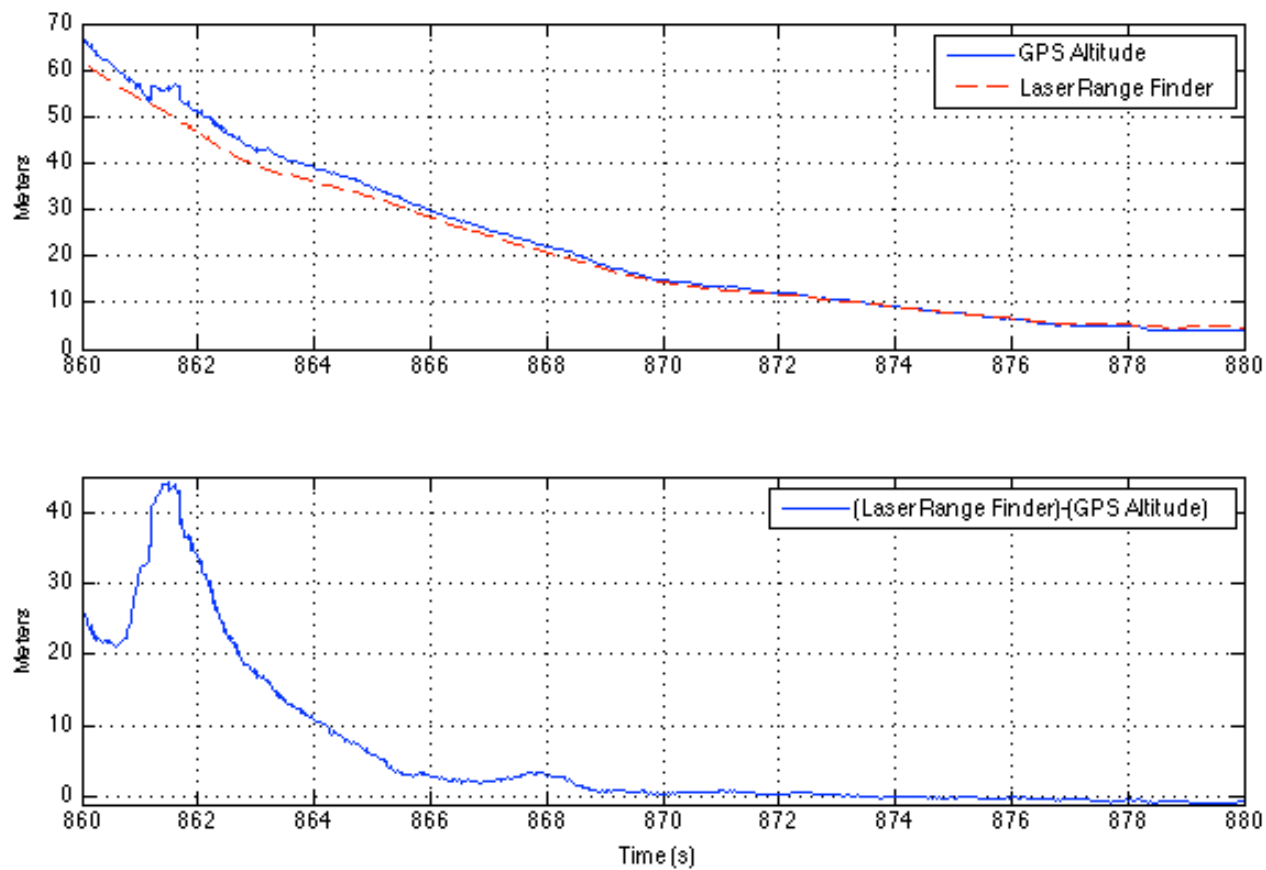

Figure 2: Comparison of GPS Altitude and Laser Range 
Figure 2 demonstrates a degradation of the measurement accuracy, as the distance gets longer. It is expected that the pitch and roll corrections are not perfect, the runway is not perfectly flat, and the GPS solution has meter-level errors.

A TOF flash LIDAR camera also uses TOF to a target to measure distance but differs from a range finder in that it, rather than perceiving the return of a single light pulse, an entire scene is captured by the receiver. These cameras have recently been evaluated for UAS 3-D navigation and SAA applications. For example, miniaturized flash LADAR, such as the SwissRanger by Mesa Imaging ${ }^{33}$, has been demonstrated for autonomous indoor navigation applications ${ }^{34}$. This system is compact with dimensions of $65 \times 65 \times 76 \mathrm{~mm}$ however, it only offers a limited range of up to 10 meters and limited field of view (FOV) of up to 66 degrees making them not as viable for outdoor navigation applications.

A scanning laser may be used for 3-D image mapping by multiple laser beams directed to, and then reflected from, a remote surface, a scan. The combination of the distance measures can then be combined to present a map of the remote surface. Soloviev and De Haag considered the feasibility of scanning LIDARS for 3-D UAS autonomous navigation for outdoor urban environments ${ }^{35}$ by modifying a 2-D scanning LIDAR, the SICK LMS-200 ${ }^{36}$ with a mounting it on a servo to provide another degree of freedom. This device offers a comparably extended range, 80 meters maximum, and field of view, 360 degrees, but at the cost of higher mass, $4.5 \mathrm{~kg}$, and power, $20 \mathrm{~W}$ typical consumption.

Hokuyo provides the URG-04LX-UG01 Scanning Laser Rangefinder ${ }^{37}$ that is small, accurate, and affordable. The effective range is from $20 \mathrm{~mm}$ to $5600 \mathrm{~mm}$ at a $1 \mathrm{~mm}$ resolution in a $240^{\circ}$ arc $\left(0.36^{\circ}\right.$ angular resolution $)$ at a scanning time of $100 \mathrm{~ms} / \mathrm{scan}$. Its size is $50 \times 50 \times 70 \mathrm{~mm}$, its weight is $160 \mathrm{~g}$, and requires $500 \mathrm{~mA}$ of current.

\section{Example of Small SWaP, Integrated Sensor}

An attractive technology that should be considered as an alternative for ranging and radar/LIDAR is Ultra Wideband radios which are low-SWaP and can simultaneously act as radar sensors, communication transceivers and peer-to-peer ranging sensors. For example, to offer robustness for navigation, MacGougan et al. ${ }^{38}$ considered GPS augmentation with a network of UWB devices to yield better accuracy during periods of poor satellite geometry, poor signal conditions, poor sky visibility, or high multipath.

Time Domain Corporation has developed an innovative multi-sensor that combines range measurement, imaging, synchronization, and data communications in a single board of sufficiently small SWaP that it is practical for integration into small autonomous vehicles and can facilitate cooperative behaviors. The UWB technology uses short pulses of energy rather than continuous sine waves with a duration of approximately $1 \mathrm{~ns}$ and bandwidth of $\sim 1.5 \mathrm{GHz}$. The characteristics of UWB yield several desirable characteristics to facilitate autonomous activities:

- Because of the accuracy of the pulses, measurement of the time of transmission between two transceivers is of sufficient accuracy that distance can be measured to within $2 \mathrm{~cm}$. Three transceivers can facilitate relative localization in 2-D. Four transceivers can facilitate relative localization in 3-D. A fixed transceiver of known location can extend to absolute localization.

- The device allows the demonstration of mono-static, bi-static and multi-static radars and can operate as a high performance imaging radar. UWB radars offer more than a GHz of RF bandwidth providing the resolution required to operate in high clutter environments. Preliminary experiments have indicated that it is also possible to analyze radar returns from a moving target and determine gait or other characteristic motions $^{39}$.

- Allows distribution of a synchronization signal to multiple locations and can maintain unit-to-unit synchronization of $50 \mathrm{ps}^{40}$.

- Transmissions can also be modulated and thereby support the transmission of data.

- Because the pulses are short in duration they are also physically short in distance. This allows the system to resolve multipath problems and operate in high clutter environments without a penalty to system accuracy or robustness of operation.

Time Domain appears to be unique in the world in the application of UWB to these 4 simultaneous capabilities in a single board. With all of its capabilities it can be utilized for a number of functions required for operation of autonomous vehicles: as an alternative to GPS for localization in cooperative vehicle behaviors; as an imaging radar for altimetry, collision avoidance, and terrain mapping; and to synchronize clocks across multiple vehicles.

These capabilities are available in Time Domain's PulsON 410 product. Supporting all capabilities, a kit may be purchased containing a single P410 module, two omnidirectional antennas, power supply, and software. One 
antenna is required for ranging applications and two are required for application as a mono-static radar. The board is $76 \times 80 \times 16 \mathrm{~mm}$, weighs $58 \mathrm{~g}$, and consumes a maximum of $4.2 \mathrm{~W}$.

If two or more vehicles are equipped with a UWB radio, distance between the radios can be determined by TOF of transmission. Data returned includes the Precision Range Measurement (PRM or the "range") and a metric called Precision Range Error Estimate (PRME.) PRME is an estimate of the statistical error (standard deviation) of the range measurement based on the shape of the underlying pulse. The minimum PRME observed has been $2.44 \mathrm{~cm}$. which substantiates Time Domain's specification of range measurement within $2.5 \mathrm{~cm}$. In an experiment, the radios were separated by various distances: $25,50,75,100,200,300,400,425$, and $450 \mathrm{ft}$. Multiple transmissions wee made at each distance. At all distances, a PRME of 2.44 was rare; most values were 5.49 . At $450 \mathrm{ft}$., nearly half of the transmissions were lost or had PRME much greater than 5.49 (e.g., 1054), thus the distance of $450 \mathrm{ft}$. was observed to be beyond the range of the radio. A small percentage of transmissions at the distances of $400 \mathrm{ft}$. and 425 $\mathrm{ft}$., returned a PRME $<5.49$; these were rejected as invalid. Under these conditions, transmissions at all distances up to $425 \mathrm{ft}$. consistently returned a PRME $<5.49$ thus, the perceived average accuracy was within $5.5 \mathrm{~cm}$ and this was consistent for all distances. A similar study was done for distances closer than $25 \mathrm{ft}$. with similar results. However, PRME represents a standard deviation accepting that some errors would be outside of this range. Examining the minimum and maximum measurement at each distance revealed a range of between 4 and $8.8 \mathrm{~cm}$ (distance did not seem to be a factor in this; the $4 \mathrm{~cm}$ range was at $425 \mathrm{ft}$.).

The PulsON 410 as delivered with its omnidirectional antenna has limited capability as a radar device. It emits period pulses and can, for example, detect movement within a circular area. It does not have any directional, scanning, or array capability. However, given a software infrastructure and better directional antenna, it has high potential as a radar device. Due to its increased bandwidth, it has distinct advantages over other technologies including robust performance in the face of multipath and the ability to reject clutter, attain high spatial resolution and discriminate between targets in close proximity to each other.

\section{Conclusion}

For an autonomous systems to behave appropriately and as expected, improved sensory perception is required to provide environmental and situation awareness necessary for cognitive decisions directing correct action. In piloted vehicles, humans acting on human sensory perception perform these functions. In autonomous vehicles, electromechanical sensors and computers must emulate the human senses. With sufficient technology, it is probable in time that this replacement will greatly exceed human capability. For small vehicles, perception equipment must not only be capable, it must be of small SWaP. Several examples of available technology were described that can facilitate visual surveying, positioning, navigating, ranging and localization, radar surveying, and image mapping. All of the examples presented were of sufficiently small SWaP to be carried and used in UAVs of less than 20 lbs. The items presented were not meant to be an exhaustive list of what is available, but rather a sample to show the trend is moving in the direction of small SWaP perception. Also the list was not presented as a comprehensive capability for autonomous perception but rather presents a view of what is available for implementation today. Much work remains to be done both in providing perceptive capability and in reducing SWaP, especially considering the goal of micro- or even nano- scale vehicles.

\section{Acknowledgments}

J. Gross was supported for this work in part by NASA WV EPSCoR and the WV Research Challenge Fund seed grant program.

\section{References}

1"Finn, A., Scheding, S., "Developments and Challenges for Autonomous Unmanned Vehicles: A Compendium", SpringerVerlag, Berlin, February 28, 2010, ISBN 978-3-642-10703-0, DOI 10.1007/978-3-642-10704-7.

${ }^{2}$ Bearth, D. P. "Amazon. com tests same-day delivery of small packages shuttled by drones," Transport Topics 4078 (2013).

${ }^{3}$ Waring, W. R., Schoepfer, V. A., Burgin, A. J., Ore, J. P., Detweiler, C., Elbaum, S., "Using Unmanned Aerial Vehicles (UAVS) to Map Sources of Groundwater in a Saline Wetland" Joint Aquatic Sciences Meeting. Portland, OR, May 2014.

4"Remote Sensing of Volcanoes and Volcanic Processes: Integrating Observation and Modelling," Geological Society of London, Volume 380, ed. Pyle, D. M., Mather, T. A., Biggs, J., 2014, ISBN 1862393621, 9781862393622.

${ }^{5}$ Wang, M. B., Chu, A., Bush, L. A., Williams, B. C., "Active detection of drivable surfaces in support of robotic disaster relief missions," Aerospace Conference, 2013 IEEE, vol., no., pp.1,13, 2-9 March 2013, DOI: 10.1109/AERO.2013.6497355

${ }^{6}$ Wingfield, N., Sengupta, S., "Drones Set Sights on U.S. Skies," New York Times, February 18, 2012, URL: http://www.nytimes.com/2012/02/18/technology/drones-with-an-eye-on-the-public-cleared-to-fly.html [cited 5 May 2014 ]. 
7"Integration of Civil Unmanned Aircraft Systems (UAS) in the National Airspace System (NAS) Roadmap," Federal Aviation Administration, 1st Edition 2013, URL: http://ntrs.nasa.gov/archive/nasa/casi.ntrs.nasa.gov/20130011508.pdf [cited 5 May 2014].

8"Kaman K-Max Helicopter," URL: http://www.kaman.com/aerospace/aerosystems/air-vehicles-mro/products-services/k-

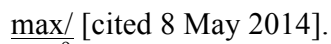

" $"$ "Sferisense 500 Hellas A20P LIDAR from Fairchild Controls," URL: http:/www.fairchildcontrols.com/ [cited 12 May 2014].

10"MQ-1B Predator," URL: http://www.af.mil/AboutUs/FactSheets/Display/tabid/224/Article/104469/mq-1b-predator.aspx [cited 12 May 2014].

${ }^{11}$ Fahlstrom, P., Gleason, T., "Introduction to UAV Systems," 4 edition, John Wiley \& Sons, Ltd, Chichester, UK, September 17, 2012, ISBN 978-1119978664.

${ }^{12}$ Srinivasan, M.V., Thurrowgood, S., Soccol, D., "Competent vision and navigation systems," Robotics \& Automation Magazine, IEEE, vol.16, no.3, pp.59,71, September 2009, DOI: 10.1109/MRA.2009.933627.

${ }^{13}$ Haiyang Chao. H., Yu Gu, Gross, J., Guodong Guo, Fravolini, M.L., Napolitano, M.R., "A comparative study of optical flow and traditional sensors in UAV navigation," American Control Conference (ACC), 2013 , pp.3858,3863, 17-19 June 2013.

${ }^{14}$ Carnie, R.; Walker, R.; Corke, P., "Image processing algorithms for UAV sense and avoid," Robotics and Automation, Proceedings 2006 IEEE International Conference on, pp.2848,2853, 15-19 May

2006. DOI: 10.1109/ROBOT.2006.1642133.

${ }^{15}$ Humphreys, T. E., et al., "Assessing the spoofing threat: Development of a portable GPS civilian spoofer," Proceedings of the ION GNSS International Technical Meeting of the Satellite Division. Vol. 55. 2008.

${ }^{16}$ Humphreys, T. "Statement on the vulnerability of civil unmanned aerial vehicles and other systems to civil GPS spoofing," University of Texas at Austin, July 18, 2012.

17"GRX2 receiver GPS receiver from Sokkia," URL: http://us.sokkia.com/products/gnss-receivers/rtk-systems/grx2-gnssreceiver [cited 5 May 2014].

18"Piksi GPS receiver from Swift Navigation," URL: http://swift-nav.com/piksi.html [cited 5 May 2014].

${ }^{19}$ Zumberge, J. F., Heflin, M. B., Jefferson, D. C., Watkins, M. M., and F. H. Webb, "Precise point positioning for the efficient and robust analysis of GPS data from large networks," Journal of Geophysical Research: Solid Earth (1978-2012) 102, no. B3, pp. 5005-5017, 1997.

${ }^{20}$ Hensley, S., Wheeler, K., Sadowy, G., Jones, C., Shaffer, S., Zebker, H., Miller T., et al., "The UAVSAR instrument: Description and first results." Radar Conference, 2008. RADAR'08. IEEE, pp. 1-6. IEEE, 2008.

${ }^{21}$ Dixon, K., "StarFire: A global SBAS for sub-decimeter precise point positioning," Proceedings of ION GNSS, 2006.

22"Omnistar Novatel Receivers," URL: http://www.omnistar.com/Support/ReceiverInformation/Novatel.aspx [Cited 14 May 2014]

${ }^{23}$ "Terrastar by Septentrio Satellite Navigation," URL: http://www.septentrio.com/terrastar [Cited 14 May 2014].

${ }^{24}$ Barbour, Neil M. "Inertial navigation sensors." Low-cost navigation sensors and integration technology (2011): 2-1.

25"Analog Devices Inc., ADIS-16488 Data Sheet", URL: http://www.analog.com/static/importedfiles/data_sheets/ADIS16488.pdf [cited: May 13, 2014].

${ }^{26}$ Gross, J., Gu, Y., Rhudy, M., Gururajan, S., Napolitano, M. "Flight Test Evaluation of Sensor Fusion Algorithms for Attitude Estimation" IEEE Transactions on Aerospace Electronic Systems, Vol. 48 Is. 3, July, 2012.

${ }^{27}$ "SBG Systems, IG-500 OEM Option", URL: http://www.sbg-systems.com/products/ig500-oem-inertial-systems [cited 13 May 2014].

28"Gladiator Technologies, LandMark Inertial Measurement Units", http://www.gladiatortechnologies.com/Products/LMRK/IMU/LandMark-IMU.html [cited 13 May 2014].

${ }^{29}$ "Epson Inertial Measurement Units", URL: http://www5.epsondevice.com/en/sensing_system/product/imu/ [cited 13 May 2014].

30"FLIR MLR-10030 miniature laser rangefinder," URL: http:/www.flir.com/cvs/cores/view/?id=56598 [cited 10 May, 2014].

31"Opti-Logic's RS400 laser rangefinder," URL: http://www.opti-logic.com/RS400-400-Yard-Laser-Rangefindingp/00002.htm [cited 13 May 2014].

${ }^{32} \mathrm{Gu}$, Y., Gross, J., Barchesky, F., Chao, H, Napolitano M., "Avionics Design for a Sub-Scale Fault-Tolerant Flight Control Test-Bed," Recent Advances in Aircraft Technology, Ramesh K. Agarwal (Ed.), ISBN: 978-953-51-0150-5.

33"Mesa Imaging, SR 4000", URL: http://www.mesa-imaging.ch/products/sr4000 [cited 13 May 2014].

${ }^{34}$ de Haag, M. U., Venable, D., and Smearcheck, M., "Use of 3D laser radar for navigation of unmanned aerial and ground vehicles in urban and indoor environments," Defense and Security Symposium, International Society for Optics and Photonics, 2007.

${ }^{35}$ Soloviev, A., de Haag, M.U., "Three-Dimensional Navigation with Scanning Ladars: Concept \& Initial Verification," IEEE Transactions on Aerospace and Electronic Systems, vol.46, no.1, pp.14,31, January 2010, DOI: 10.1109/TAES.2010.5417145
36" Sick
Automation,
LMS-200,"
URL:
http://www.sick-

automation.ru/images/File/pdf/LMS\%20Technical\%20Description.pdf [cited 13 May 2014].

37,"Hokuyo URG-04LX-UG01 Scanning Laser Rangefinder", URL: https://www.hokuyoaut.jp/02sensor/07scanner/urg 041x.html, [cited 13 May 2014]. 
${ }^{38}$ MacGougan, G., O’ Keefe, L., Klukas, R. “Tightly-Coupled GPS/UWB Integration,” Journal of Navigation, Vol. 63, Is. 01, pp. 1-22, Jan. 2010.

${ }^{39}$ Petroff, A., "A practical, high performance Ultra-Wideband radar platform," Radar Conference (RADAR), 2012 IEEE, pp. 0880,0884, 7-11 May 2012, DOI 10.1109/RADAR.2012.6212261.

40"Time Domain's Ultra Wideband (UWB) - Definition and Advantages," URL: http://www.timedomain.com/whitepapers/320-0294B\%20Time\%20Domain\%27s\%20UWB\%20Definition\%20\&\%20Advantages.pdf [cited 10 May 2014 ]. 